DOSSIÊ TEMÁTICO: Perspectivas para pensar as cidades: infâncias, educação, democracia e justiça

d०1 https://doi.org/10.22481/praxisedu.v16i40.6899

\title{
IL DIRITTO DI GIOCARE: UNA NECESSITÀ PER I BAMBINI, UN POTENZIALE PER SCUOLA E LA CITTÀ
}

\author{
O DIREITO DE BRINCAR: UMA NECESSIDADE PARA AS CRIANÇAS, UMA \\ POTENCIALIDADE PARA A ESCOLA E A CIDADE
}

THE RIGHT TO PLAY: A NECESSITY FOR CHILDREN, A POTENTIAL FOR SCHOOL AND THE CITY

\section{EL DERECHO AL JUEGO: UNA NECESIDAD PARA LOS NIÑOS, UN POTENCIAL PARA LA ESCUELA Y LA CIUDAD}

\author{
Francesco Tonucci \\ Instituto de Ciências e Tecnologias da Cognição - Itália \\ Conselho Nacional de Pesquisa - Itália
}

\begin{abstract}
Astratto: Il principale interrogativo affrontato nell'articolo: perché e come il gioco dei bambini dovrebbe essere considerato un importante parametro nelle politiche urbane? Una possibile risposta a questo problema si trova in tutto il testo. Voglio iniziare tratteggiando la più recente evoluzione (o piuttosto involuzione) della struttura e della organizzazione urbana enfatizzando come questa definisce un ambiente che è proibito ed ostile per i bambini a meno che siano accompagnati dagli adulti; riassumerò l'impatto di un simile ambiente sul gioco e quindi sullo sviluppo dei bambini, accentuando le diverse caratteristiche della mobilità spaziale nei bambini e negli adulti, e l'estremo pericolo di isolare i bambini dall'esperienza del pericolo che costituisce una caratteristica paradossale della educazione di oggi. Proporrò un'alternativa radicale alla corrente politica urbana in cui i bambini e il loro gioco sono il principale focus, piuttosto che un marginale e non conosciuto problema da essere considerato solo come un ripensamento, e presenterò alcuni casi concreti nei quelli una simile alternativa è stata messa in pratica da bambini e politici insieme, e i suoi effetti sono stati testati e valutati. In conclusione esperienze simili sembrano giustificare una moderata quantità di speranza per il futuro delle nostre città, nonostante le loro preoccupanti condizioni attuali.
\end{abstract}

Parole chiave: bambini, scuola, diritto a giocare, citá

Resumo: A principal questão abordada neste artigo é: por que e como a brincadeira infantil deve ser considerada um parâmetro importante nas políticas urbanas? Uma possível resposta para este problema é encontrada ao longo do texto. Gostaria de começar desenhando a evolução mais recente (ou melhor, involução) da estrutura e organização urbana, enfatizando como ela define um ambiente que é proibido 
e hostil às crianças, a menos que sejam acompanhados por adultos; vou resumir o impacto desse ambiente no jogo e, portanto, no desenvolvimento das crianças, acentuando as diferentes características da mobilidade espacial em crianças e adultos, e o extremo perigo de isolar as crianças da experiência do perigo. Proporei uma alternativa radical à política urbana na qual as crianças e seu jogo são o foco principal, em vez de um problema marginal e não conhecido para ser considerado apenas como uma reflexão posterior, e apresentarei alguns casos concretos naqueles que tal alternativa foi colocada em prática por crianças e políticos juntos, e seus efeitos foram testados e avaliados. Em conclusão, experiências semelhantes parecem justificar uma quantidade moderada de esperança para o futuro de nossas cidades, apesar de suas preocupantes condições atuais.

Palavras chave: Crianças; Direito de brincar; Escola; Cidade.

\begin{abstract}
The main question addressed in this article is: why and how should child play be considered an important parameter in urban policies? A possible answer to this problem is found throughout the text. I would like to begin by drawing the most recent evolution (or rather involution) of urban structure and organization, emphasizing how it defines an environment that is forbidden and hostile to children, unless they are accompanied by adults; I will summarize the impact of this environment on the game and therefore on the development of children, accentuating the different characteristics of spatial mobility in children and adults, and the extreme danger of isolating children from the experience of danger. I will propose a radical alternative to urban policy in which children and their play are the main focus, rather than a marginal problem and not known to be considered only as a later reflection, and I will present some concrete cases in those where such an alternative was put into practice by children and politicians together, and its effects were tested and evaluated. In conclusion, similar experiences seem to justify a moderate amount of hope for the future of our cities, despite their worrying current conditions.
\end{abstract}

Keywords: Childreen, City, School, right to play

Resumen: La pregunta principal que se aborda en este artículo es: ¿por qué y cómo se debe considerar el juego infantil un parámetro importante en las políticas urbanas? Una posible respuesta a este problema se encuentra en todo el texto. Me gustaría comenzar dibujando la evolución más reciente (o más bien involucionada) de la estructura y organización urbana, haciendo hincapié en cómo define un entorno que está prohibido y hostil a los niños, a menos que vayan acompañados de adultos; Resumiré el impacto de este entorno en el juego y, por lo tanto, en el desarrollo de los niños, acentuando las diferentes características de la movilidad espacial en niños y adultos, y el peligro extremo de aislar a los niños de la experiencia del peligro. Propondré una alternativa radical a la política urbana en la que los niños y su juego sean el foco principal, más que un problema marginal y no se sepa que sólo se considere como una reflexión posterior, y presentaré algunos casos concretos en aquellos en los que los niños y los políticos pusieron en práctica una alternativa de este tipo, y sus efectos fueron probados y evaluados. En conclusión, experiencias similares parecen justificar una cantidad moderada de esperanza para el futuro de nuestras ciudades, a pesar de sus preocupantes condiciones actuales.

Palabras clave: ciudad, derecho al juego, escuela, niños

Chi governa una città prende decisioni che riguardano apparentemente aspetti concreti e quotidiani della vita delle persone, come i trasporti, le abitazioni, i servizi. Di fatto queste scelte intervengono su un problema di fondo che riguarda la felicità dei cittadini. La città può 
assumere problemi come il traffico, la velocità degli spostamenti e la difesa degli spazi pubblici come prioritari e sacrificare ad essi i bisogni di comunicazione, di scambio e di serenità specialmente per i cittadini più deboli. Può al contrario ritenere prioritario assicurare il diritto di gioco e di mobilità a bambini ed anziani, ricercare la sicurezza nella solidarietà e nella partecipazione, difendere l'estetica urbana come bene irrinunciabile. Diceva il sindaco di Curitiba, in Brasile, che aveva potuto dotare tutti i quartieri della città di biblioteche pubbliche e dotarle di una buona quantità di libri rinunciando a costruire un chilometro di autostrada. A dimostrazione del fatto che spendere per i bambini non è un costo reale ma un vero investimento produttivo anche dal punto di vista economico, Kofi Annan, il presidente delle Nazioni Unite l'8 maggio 2002 a New York aprendo la Sessione Speciale dell'ONU per l'Infanzia, chiudeva il suo intervento dicendo: "Come potremo fallire, soprattutto ora che sappiamo che ogni dollaro investito nel migliorare le condizioni dell'infanzia ha un ritorno per tutta la società di ben 7 dollari?" Queste sono scelte di filosofia di governo della città.

L'obiettivo principale di questo articolo: permettere ai bambini di giocare secondo le loro necessità (e non secondo quanto permesso da una città adattata alle sole necessità dell'adulto) garantisce cittadini adulti più sani, più sereni e più produttivi. In alter parole, il gioco è indubbiamente un aspetto chiave delle politiche urbane. Ma perché è così?

Questo è il principale interrogativo affrontato nell'articolo: perché e come il gioco dei bambini dovrebbe essere considerato un importante parametro nelle politiche urbane? Una possibile risposta a questo problema è presentata nelle seguenti pagine: voglio iniziare tratteggiando la più recente evoluzione (o piuttosto involuzione) della struttura e della organizzazione urbana (cf.1), enfatizzando come questa definisce un ambiente che è proibito ed ostile per i bambini a meno che siano accompagnati dagli adulti (cf. 2); riassumerò l'impatto di un simile ambiente sul gioco e quindi sullo sviluppo dei bambini (cf. 3), accentuando le diverse caratteristiche della mobilità spaziale nei bambini e negli adulti (cf. 4), e l'estremo pericolo di isolare i bambini dall'esperienza del pericolo che costituisce una caratteristica paradossale della educazione di oggi (cf. 5). Proporrò un'alternativa radicale alla corrente politica urbana in cui i bambini e il loro gioco sono il principale focus, piuttosto che un marginale e non conosciuto problema da essere considerato solo come un ripensamento (cf. 6), e presenterò alcuni casi concreti nei quelli una simile alternativa è stata messa in pratica da bambini e politici insieme, e i suoi effetti sono stati testati e valutati (cf. 7). In conclusione esperienze simili sembrano giustificare una moderata quantità di speranza per il futuro delle nostre città, nonostante le loro preoccupanti condizioni attuali (cf. 8). 


\section{Lo sviluppo delle città: una breve analisi}

La città moderna nasce rompendo lo schema medioevale che divideva il castello dal borgo e creava fra l'uno e l'altro un rapporto gerarchico. Nasce intorno ad una piazza, in uno spazio condiviso, pur nella diversità dei ceti e delle condizioni. Sulla piazza si affacciano il palazzo del governo, la cattedrale, la caserma della guarnigione, nella piazza si svolge il mercato. La città è il luogo di incontro e di scambio. Lo spazio urbano è condiviso, non ci sono quartieri diversi per i diversi livelli sociali: nelle strade della città il palazzo dei nobili, spesso opera di grandi architetti, sorge a fianco delle umili casette degli artigiani. Questa alternanza costruisce un ritmo urbanistico che fa belle molte città europee.

Nelle ultime decine di anni, dopo la seconda guerra mondiale, sembra quasi che la città sia tornata al modello medioevale: il centro storico ricco si svuota, diventa sede di attività commerciali e terziarie, e viene circondato da un nuovo borgo, più squallido e duro di quello antico, la periferia povera e a volte misera, di case anonime o di baracche, che dipende dalla città ricca per la sua sopravvivenza. Il centro ricco è difeso dalla polizia, dalle telecamere fisse e da innumerevoli ditte di difesa privata.

La città ha rinunciato ad essere luogo di incontro e di scambio e ha scelto come nuovi criteri di sviluppo la separazione e la specializzazione. La separazione e specializzazione degli spazi e delle competenze: posti diversi per persone diverse, posti diversi per funzioni diverse. Il centro storico per le banche, i negozi di lusso, il divertimento; la periferia per dormire. Poi ci sono i luoghi dei bambini, l'asilo nido, il parco giochi, la ludoteca; i luoghi dei vecchi, l'ospizio, il centro anziani; i luoghi della conoscenza, dalla scuola dell'infanzia all'Università; i luoghi specializzati per le compere, il supermercato, il centro commerciale. Poi c'è l'ospedale, il luogo della malattia ${ }^{1}$ (BOZZO, 1995).

\section{Una città senza bambini}

Una volta non si vedeva l'ora di uscire di casa, perché tutto quello che interessava di più era fuori. La casa era il luogo fondamentale delle sicurezze, dei bisogni primari, dei compiti per casa. Ma bisognava uscire per incontrare gli amici, per giocare, per andare al bar, al cinema,

\footnotetext{
${ }^{1}$ Mumford chiama gli ospedali «I magazzini delle malattie» (MUMFORD, 1945).
} 
in biblioteca. E se c'erano pericoli, come c'erano, bisognava stare attenti, così dicevano i nostri genitori.

Oggi non si vede l'ora di tornare a casa, perché la casa è il luogo del riposo, della cultura, degli affetti, della comunicazione. A casa abbiamo cibi surgelati che durano mesi, abbiamo la biblioteca, la raccolta dei $\mathrm{CD}$, i film preferiti, la possibilità di parlare per telefono o di scambiarci messaggi e foto per internet o per cellulare. La casa non è più una parte importante ma insufficiente della più vasta realtà della città, ma riassume in sé la città stessa. Non è più una parte di un complesso ecosistema, ma tende essa stessa all'autosufficienza, altra caratteristica importante e inquietante della città moderna. All'autosufficienza tendono le varie parti della città, dalla casa al Centro commerciale.

Una volta comprare significava compiere un percorso, entrare in posti diversi, incontrare varie persone, ogni giorno le stesse, tanto da poter riprendere da un giorno all'altro una confidenza, un racconto o scambiarsi l'ultima notizia. Oggi per comprare si effettua un trasferimento in un'altra zona della città, o meglio in un'altra piccola ed efficiente città, dove si può comprare tutto: il Centro commerciale. Città con parcheggio garantito e quindi senza macchine, con strade e piazzette, sicura per i bambini, per i quali sono spesso pensati spazi dedicati e assistiti; dove si può mangiare, fare operazioni bancarie, andare dal parrucchiere e naturalmente comprare. Un bel posto, per molte famiglie, dove darsi un appuntamento per passare insieme il fine settimana. Il degrado rende la città invivibile, e noi ci difendiamo costruendo luoghi sicuri, protetti, dove passare tranquilli il nostro tempo libero.

Questa è una tendenza costante nella città di oggi, coerente con la logica della separazione e della specializzazione: creare servizi, strutture sempre più indipendenti e autosufficienti. Questo avviene per l'ospedale, per lo stadio, per i grandi musei, per il campus universitario (GERMANOS 1995; TAYLOR ET AL. 1998; BAUMANN 2000).

Inoltre la città negli ultimi decenni è diventata uno spazio di fatto riservato alle automobili. Queste hanno invaso gli spazi pubblici della città privatizzandoli, togliendoli al possibile uso di chi si muove a piedi o in bicicletta. Le strade e le piazze sono luoghi di transito e di sosta di mezzi privati e non più spazi pubblici che fanno di un insieme di case private una città. Alle auto spetta sempre il livello zero, sono i pedoni che, per attraversare la strada, devono scendere dal marciapiedi, scendere nei sottopassaggi o arrampicarsi sui sovrappassi pedonali. Ai pedoni, che possono anche essere piccoli, anziani, portatori di handicap, carichi delle borse della spesa, con un bambino in braccio o nel passeggino, tocca il percorso più lungo e più faticoso. Alle automobili, che pure hanno il motore, tocca sempre la strada piana, il livello zero. 
Le macchine impongono alle città la loro logica, la loro estetica, la loro "musica". Le nostre grandi città debbono ospitare, oltre alle auto, i segnali stradali verticali e le strisce in terra sui selciati antichi che inquinano visivamente i monumenti e i centri storici (GARLING, VALSINER, 1985).

Tutto questo ed altro ancora è successo, ed è successo in tempo molto breve. Perché la città, la sua amministrazione ha scelto come cittadino prototipo un cittadino maschio, adulto, lavoratore e automobilista. Ha adeguato la città alle sue esigenze, ha cercato di corrispondere alle sue richieste garantendo così il consenso elettorale del cittadino forte. Ha così tradito le esigenze e i diritti di chi non è maschio, non è adulto, non è lavoratore, non è automobilista. Con la conseguenza che queste città sono città senza bambini. In questa città il bambino non può vivere alcune esperienze fondamentali per il suo sviluppo come l'avventura, la ricerca, la scoperta, il rischio, il superamento dell'ostacolo e quindi la soddisfazione, l'emozione (LYNCH, 1979)2 . Non può giocare. Queste esperienze hanno bisogno di due condizioni fondamentali che sono scomparse: il tempo libero e uno spazio pubblico condiviso. È difficile per il bambino uscire di casa da solo, cercarsi dei compagni ed andare in un posto adeguato per giocare con loro. Le difficoltà ambientali, vere o presunte, hanno convinto i genitori che questa città non permette ad un bambino di sei, dieci anni di uscire da solo e quindi quello che era il suo tempo libero si è trasformato in tempo organizzato e dedicato a diverse attività, in casa o fuori casa, rigorosamente programmate e di solito a pagamento. Da una parte quindi la televisione, la play station, internet, dall'altra i vari corsi pomeridiani di sport, di arte e di lingue straniere (MARILLAUD, 1991).

E per giocare? Per giocare i genitori accompagnano il bambino al giardinetto attrezzato vicino a casa oppure lo accompagnano a casa di amici oppure invitano a casa i suoi amichetti. Lo aspettano e lo vigilano.

\section{All'inizio viene il gioco}

Vedendo i nostri bambini così ben vestiti, ben nutriti e capaci di manipolare apparati così complessi, che parlano lingue straniere così precocemente, che praticano uno sport per

\footnotetext{
${ }^{2}$ In questa analisi critica non dovremmo mai dimenticare quali significativi miglioramenti nelle condizioni di vita (dei cittadini in generale e dei bambini in particolare) caratterizzano le società contemporanee, rispetto ai precedenti sistemi sociali ed economici. Tuttavia, è proprio alla luce di tali progressi che non possiamo accettare passivamente la violazione dei diritti primari descritta in questo saggio, che è principalmente subordinato a interessi egoistici ed economici.
} 
davvero, rispettandone le regole e conoscendone le astuzie fin dai primi anni, spesso noi adulti pensiamo che sono proprio fortunati. Che loro possono fare molte cose che a noi non erano concesse, un po' perché non c'erano, un po' perché non c'erano i soldi e poi perché i nostri genitori non capivano queste cose.

È vero, i nostri bambini non possono correre, scatenarsi, sporcarsi, fare cose strane e a volte proibite, come abbiamo fatto noi alla loro età, ma ne vale la pena. Oggi sanno molte più cose, sono più intelligenti.

Una bambina di 10 anni del Consiglio dei bambini di Roma, discutendo sul diverso peso che hanno nella vita dei bambini il diritto al gioco e il diritto all'istruzione, riassume bene questo pensiero adulto quando dice: "Per gli insegnanti lo studio è importante perché ci prepara per il futuro, invece giocare si gioca solo da bambini e per questo non conta niente".

Contro questo atteggiamento disattento degli adulti, che hanno dimenticato il valore delle loro esperienze infantili, il gioco assume invece una importanza fondamentale sia nella storia degli uomini che nella storia personale di ogni donna e di ogni uomo.

Huizinga, alla fine degli anni Trenta, ha analizzato l'attività ludica come anticipatrice e fondante della cultura e delle culture umane: «Il gioco è più antico della cultura, perché il concetto di cultura, per quanto possa essere definito insufficiente, presuppone in ogni modo convivenza umana, e gli animali non hanno aspettato che gli uomini insegnassero loro a giocare»(HUIZINGA, 1939, p. 3). E Callari Galli, quasi a riassumere il lungo dibattito, scriveva: «L'attività ludica è presente in tutti i gruppi umani anche se la gamma delle sue variazioni è amplissima; è maggiormente presente in alcune età anche se in generale accompagna l'intero arco della vita di un individuo e si differenzia a seconda del sesso, dell'occupazione, della ricchezza; segue i mutamenti della società presentando tuttavia stupefacenti permanenze e grande continuità temporale. Per alcuni aspetti e in determinate circostanze è un'attività che sembra essere distaccata rispetto a interessi materiali e contingenti, avente uno scopo in sé, delimitato in termini di tempo e di spazio, secondo regole liberamente accettate e assolutamente vincolanti. E tutti i giochi sembrano richiedere la comprensione da parte dei partecipanti del fatto che ciò che si fa non è ciò che sembra» ${ }^{3}$.

Così come si ipotizza che il gioco precede e fonda la cultura umana, con certezza si può dire che il gioco fonda lo sviluppo individuale di ogni donna e di ogni uomo.

\footnotetext{
${ }^{3}$ Introduzione a Sutton-Smith (2002) p. 14.
} 
Nella descrizione che dello sviluppo fa la ricerca scientifica è proprio nei primi giorni, nei primi mesi e nei primi anni che lo sviluppo è più rapido, è lì, subito alla nascita che avviene l'esplosione, non intorno ai sei anni con l'inizio della cosiddetta età della ragione, come sembra credere ancora la maggioranza degli adulti, dei genitori e degli insegnanti. Prima che un bambino entri per la prima volta in una aula scolastica, le cose più importanti sono già successe: gli apprendimenti più importanti, le fondamenta sulle quali tutta la conoscenza successiva dovrà costruirsi o sono già acquisiti o difficilmente potranno essere recuperati.

Ma come si può spiegare un fenomeno così sconcertante? Nei primi anni di vita non ci sono insegnanti, non si usano materiali didattici e non si fanno programmi, e allora a cosa possiamo attribuire il merito di una crescita così importante? Mi pare che non possiamo non riconoscere in questo (anche) il ruolo della più significativa attività di questi primi anni: il gioco.

Perché questa attività infantile ha un potere così grande?

Il bambino vive nel gioco un'esperienza rara nella vita dell'uomo, quella di confrontarsi da solo con la complessità del mondo. Lui, con tutte le sue curiosità, con tutto quello che sa e che sa fare, e con tutto quello che non sa e che desidera sapere, di fronte al mondo con tutti i suoi stimoli, le sue novità, il suo fascino. E giocare significa ritagliarsi ogni volta un pezzetto di questo mondo: un pezzetto che comprenderà un amico, degli oggetti, delle regole, uno spazio da occupare, un tempo da amministrare, dei rischi da correre. Con una libertà totale, perché quello che non si può fare, si può inventare. Con una sostanziale libertà rispetto ai vincoli e alle limitazioni della realtà: quello che non c'è si può immaginare, quello che non è possibile si può inventare. "Io ero un principe medioevale" può affermare un bambino e da quel momento, se gli altri accettano la finzione, ci si trasferisce in un'altra epoca e si rispettano le sue regole, le sue condizioni: la scopa sarà per tutti un cavallo, il righello un pugnale, la capanna o l'albero o il sottoscala un castello ${ }^{4}$. Si realizza così il significato letterale di auto-nomia: capacità di governarsi con proprie leggi, capacità di darsi, di inventare nuove regole.

È proprio grazie a questa complessità che nei primi anni si realizzano gli apprendimenti di gran lunga più importanti di tutta la vita dell'uomo. E nessun adulto potrà prevedere o misurare la quantità di apprendimento di un bambino che gioca e questa sarà sempre diversa e probabilmente superiore a quella che noi potremo immaginare. Nessuno potrà programmare 0 accelerare questo processo, pena impedirlo o impoverirlo. Forse sarebbe più utile per i bambini

4 "È questo atteggiamento non letterale che permette al gioco di essere sganciato dalle sue conseguenze: in effetti ciò permette al gioco di essere gioco". (GARVEY, 1979, p. 15 manca anno edizione originale). 
che queste conoscenze rimanessero nascoste perché, conoscendole, potrebbe venire in mente agli adulti di aiutarli, di sostenerli con opportuni insegnamenti e materiali didattici, come già si è iniziato a fare con i cosiddetti "giocattoli intelligenti". Verrebbe a mancare così la condizione principale di questo prodigio e cioè che gli adulti "lascino fare", "lascino giocare" i bambini. Il giocare del bambino, prima e fuori della scuola è "perdere tempo", è perdersi nel tempo, è incontrarsi con il mondo in un rapporto eccitante, pieno di mistero, di rischio, di avventura. $\mathrm{E}$ il motore è il più potente che l'uomo conosca: il piacere. È per questo che un bambino per giocare può anche dimenticarsi di mangiare. Il gioco libero e spontaneo del bambino assomiglia alle esperienze più alte e straordinarie dell'adulto come quelle della ricerca scientifica, della esplorazione, dell'arte, della mistica; le esperienze appunto di quando l'uomo si trova di fronte alla complessità, di quando trova di nuovo la possibilità di lasciarsi trasportare dal grande motore del piacere (BATESON, 1956; BENCIVENGA, 1995; BRUNER, 1976).

Le proposte educative, pure necessarie, si muovono invece ad un livello più basso, meno stimolante e per questo meno produttivo. Nella proposta educativa l'allievo viene privato della eccitazione dell'incontro con la complessità e del brivido di ritagliarsene autonomamente una parte. È l'adulto che propone all'allievo una porzione di quel mondo complesso, tale che l'attività richiesta produca con sicurezza e nei tempi previsti gli apprendimenti voluti. Quel pezzo di mondo perde tutto il fascino e il mistero, diventa incomprensibile, così staccato da tutto il resto, e serve solo per imparare. Per essere più sicuri del risultato gli adulti educatori spesso sostituiscono la complessità del mondo reale con quella più controllabile della proposta didattica, dell'esercizio, del libro di testo. Il controllo è così assoluto, ma in genere il risultato è povero, quasi sempre inferiore alle aspettative e contraddittorio: mentre impara l'allievo rifiuta quello che gli insegnano, non lo fa suo, non si modifica grazie a quello. Nasce un apprendimento parallelo, che serve solo a scuola, fino all'ultimo tema in classe, fino all'ultimo concorso e poi basta. La scuola, con questa sua semplificazione, con la sicurezza della sua programmazione, ha perso completamente il rapporto con il piacere e deve ricorrere ad un motore molto meno potente ed efficace, quello del dovere.

Forse per questo legame al dovere lo studio prima e il lavoro poi vengono accreditati come comportamenti "etici" mentre il gioco e il giocare, legati profondamente al piacere vengono banalizzati come infantili, improduttivi, opzionali. Forse per questo il gioco dei bambini è stato nel recente presente sollevato alla "dignità" di consumo e quindi, in qualche modo affrancato dalla sua peculiari caratteristiche di inutilità e gratuità. 
Forse per questo il gioco dei bambini gode e soffre dei privilegi e delle emarginazioni di cui godono e soffrono i filosofi, gli artisti, i ricercatori, gli scienziati. Anche per questi la società non tollera che la loro attività sia gratuita, inutile e legata al principio del piacere.

\section{Andrei adagio adagio verso una fontana}

Proviamo a seguire due percorsi: quello di un bambino che si muove tenuto per mano da un adulto e quello di un bambino che si muove da solo.

Nel primo caso il bambino non prende alcuna decisione, o meglio, non può prendere alcuna decisione. Se prova a fermarsi, a raccogliere qualcosa, a modificare il percorso, di solito viene richiamato con le consuete esortazioni: "Non ti fermare sempre!", "Dai che facciamo tardi!", "Non toccare che ti sporchi!" Per l'adulto lo spostarsi è un trasferimento da un punto di partenza ad uno di arrivo, nel tempo più breve possibile, senza incidenti o imprevisti. Non è un caso che i sistemi considerati più efficienti per lo spostamento siano la metropolitana, l'autostrada e l'aereo. Sistemi che con meno "distrazioni" possibili fanno raggiungere nel tempo più breve il luogo di destinazione. Questo è l'obiettivo del viaggio, l'interesse principale dell'adulto. Il percorso è una perdita di tempo, va compresso sempre di più. Si è disposti a spendere cifre incredibili per ridurre di trenta minuti il percorso in treno di quattro ore fra Roma e Milano!

Nel secondo caso il bambino è da solo, può decidere, anzi non ha rimedio alla necessità di decidere, altrimenti deve tornare a casa immediatamente. Ad ogni passo deve prendere diverse decisioni: se proseguire, se cambiare direzione, se fermarsi a osservare qualcosa, se raccogliere qualche oggetto che lo interessa. E ogni decisione che prende comporta delle conseguenze: se si ferma dovrà tener conto del tempo altrimenti farà tardi, se raccoglie qualcosa dovrà poi nasconderlo perché la mamma non vuole, se cambia direzione dovrà fare attenzione a prendere nota di alcuni indicatori che possano aiutarlo al ritorno a ritrovare il cammino. Va notato che gli indicatori utili per il ritorno occorre prenderli a rovescio, così come si presenteranno tornando indietro! Ognuna di queste operazioni è complessa, ricca di elementi cognitivi, spaziali, affettivi. In un semplice trasferimento dalla sua casa al negozio di frutta il bambino può scoprire sassi di vari colori, foglie secche, piccoli animali, fogli di riviste con strane fotografie (a volte non "adatte" per lui), può cambiare il percorso facendo attenzione a non perdersi, deve poi spiegare alla fruttivendola cosa deve comprare, deve pagare, accettare o rifiutare il fico che gli regala, tornare a casa. Quante cose avrà da raccontare questo bambino 
alla mamma! Per il bambino spostarsi è un percorso, un itinerario fatto di tanti punti intermedi ognuno dei quali è più importante del punto di arrivo che rappresenta solo la fine delle scoperte e dell'avventura. Esattamente il contrario dell'adulto.

«Se avessi» said the little prince to himself «53 minuti da spendere andrei adagio adagio verso una fontana» (SAINT-EXUPÉRY, 1943).

Questa del modo di muoversi dell'adulto e del bambino è un bella metafora per comprendere due filosofie di vita diverse, due modi di affrontare non solo lo spazio, ma il tempo, le relazioni sociali, l'apprendimento, e il gioco.

Per giocare i bambini devono avere cinquantatré minuti da spendere, un tempo libero, da usare come vogliono. Nel quale poter provare l'insostenibile sensazione della noia, del non saper cosa fare e del dover fare qualcosa per uscirne, con un compagno, con una idea, con un giocattolo, inventando qualcosa. Un tempo nel quale l'adulto non debba, non voglia mettere la sua autorità o la sua competenza o anche solo la sua vigilanza protettiva. Un tempo che il bambino possa spendere.

E poi occorre una fontana verso cui andare. Non è importante arrivare alla fontana, ma compiere un percorso, una esplorazione, una ricerca. Per questo lo spazio che serve ai bambini per giocare deve essere uno spazio non limitato, non chiuso, non povero, non fatto apposta per giocare.

Un bambino di pochi mesi non dovrebbe essere tenuto dentro il box, meglio lasciarlo su una vecchia coperta. Dentro il box sembra felice, con tutti i suoi giochini a portata di mano, ma non può uscire, non può rischiare...Dalla coperta può gattonare fuori, fino a girare dietro l'angolo e scomparire per un attimo dall'occhio vigile della mamma: la prima avventura! E poi un giorno dovrà poter uscire dalla porta, incontrarsi con un altro bambino sul pianerottolo, giocare con lui sulle scale. Scendere insieme nel cortile, poi sul marciapiedi. Più tardi andare con gli altri bambini nel giardinetto vicino a casa e poi più lontano, attraversare la strada, andare nella piazza, esplorare luoghi nuovi, più lontani. Osservare gli adulti, imitarne le attività, spiarne i comportamenti spesso strani del lavoro, della lite, dell'amore. Per giocare un bambino ha bisogno di una città, perché l'ambiente di gioco deve poter crescere con lui (PREZZA ET AL. 2000; CHAWLA 2001).

Allora vanno considerati inadeguati e controproducenti quei giardini per bambini, tutti uguali, tutti rigorosamente livellati e sempre dotati di arredi stereotipati come le altalene, gli scivoli, le giostrine o i più moderni praticabili di legno. Sono luoghi che rimarranno sempre 
uguali e dove i bambini dovranno sempre andare accompagnati e vigilati dai loro genitori (ADER, JOUVE, 1991).

Diceva bene l'allora presidente del Consiglio italiano Romano Prodi al primo Forum Internazionale delle città a misura di bambino a Napoli nel 1997: "Non basta più dare ai bambini servizi per l'infanzia, dobbiamo ridare loro le città".

\section{Il rischio di non incontrare rischi}

Una differenza importante fra l'essere bambini di quaranta, cinquanta anni fa e di oggi è che una volta spesso i genitori non c'erano. In quei periodi di assenza di controllo, durante i quali tuttavia valevano precise regole di tempo, di spazio e di cose lecite o meno, i bambini potevano vivere le loro esperienze più importanti, potevano incontrare le novità, gli ostacoli, le avventure, i rischi. In quei periodi, o per lo meno, specialmente in quei periodi i bambini crescevano. Sperimentavano le strategie di socializzazione con gli altri bambini: le vergognose sottomissioni per essere accettati dai grandi, le strane attività richieste per giocare con bambini dell'altro sesso, la forza che serviva per vincere e quella necessaria per perdere. Sperimentavano le proprie capacità sottoponendole ogni volta a prove più impegnative sotto l'occhio vigile degli altri. Sperimentavano la soddisfazione della prova superata e la frustrazione dell'insuccesso. Così si diventava grandi. La sera, stanchi, sporchi e graffiati, dopo i rimproveri, c'erano tante cose da raccontare a casa!

Oggi gli adulti ci sono sempre. Questa è la grande differenza. Se non è un genitore è l'insegnante oppure l'istruttore o il catechista o l'operatore della biblioteca o della ludoteca. Se l'adulto è presente il bambino non può correre rischi (l'adulto è lì apposta perché questo non succeda) e non può neppure esplorare, scoprire, sorprendersi (l'adulto è lì apposta per spiegare, per anticipare, per rispondere) (BLAKELY 1994; AMERIO 1999). Infatti i bambini non hanno più nulla da raccontare perché tutto quello che hanno vissuto nella giornata era alla presenza di altri, di testimoni, di controllori. L'impossibilità di sperimentare l'ostacolo, la prova, quando i bambini li sentono necessari, porta ad accumulare un'esigenza, una voglia di emozioni e di rischio che tenderanno ad essere soddisfatte tutte insieme quando finalmente l'autonomia sarà raggiunta, quando il ragazzino, la ragazzina, avranno in tasca le chiavi di casa o sotto il sedere un motorino. E potrebbe essere pericoloso. Anche questa è una lettura possibile delle difficoltà e dei drammi dell'adolescenza. 
Questa scomparsa della esperienza del rischio dalla vita infantile non è presente solo nell'ambito più evidente delle abilità fisiche e delle esperienze concrete. Possiamo riscontrarla per esempio anche nello sviluppo cognitivo, in quello sociale e in quello emotivo.

Se il bambino non può esplorare, cercare, indagare, difficilmente può trovarsi di fronte a problemi sconosciuti con il desiderio di affrontarli e risolverli da solo, difficilmente potrà vivere l'emozione di trovare soluzioni nuove, non ortodosse, eppure efficaci. Piaget diceva che le conoscenze che non si costruiscono non servono, ma se l'adulto è sempre presente difficilmente si può evitare di chiedere il suo aiuto e di accettare le sue risposte, le sue verità (TONUCCI, 1995). La scuola si basa quasi esclusivamente su questa relazione fra chi sa e per questo insegna e chi non sa e per questo deve imparare.

Nel campo sociale per un bambino di oggi è quasi impossibile vivere il rischio e l'emozione di incontrare bambini sconosciuti con i quali sperimentare le delicate strategie dell'approccio, della conoscenza e qualche volta del rifiuto. I suoi compagni di gioco sono quasi esclusivamente i suoi compagni di classe, i suoi compagni dei corsi pomeridiani o i figli degli amici dei genitori: amicizie controllate e controllabili da parte degli adulti. Non sarà facile per un bambino o una bambina che non hanno mai scelto i compagni di gioco scegliere da grande una compagna o un compagno per la vita.

Nel campo delle emozioni c'è spesso da parte degli adulti una preoccupazione di assecondare e se possibile anticipare i desideri e le richieste dei figli. Viene così a mancare il fondamentale rapporto fra le tante cose ed esperienze desiderate e sognate e le poche che si possono realizzare con grande felicità e gratitudine. Stupisce leggere di reazioni spropositate di adolescenti che arrivano a ferire o ad uccidere di fronte ad un rifiuto o ad una sconfitta, ma evidentemente per loro la frustrazione non era una esperienza prevista ed elaborata ${ }^{5}$.

\footnotetext{
5 È interessante notare come l'esperienza del rischio sia indicata come un valore positivo nello studio di una delle malattie infantili più gravi come la leucemia. Greaves, genetista epidemiologo inglese, che studia i fattori che favoriscono la leucemia ha documentato come l'essere esposti nei primi anni ad infezioni comporta uno sviluppo del sistema immunitario capace, almeno in parte, di diminuire il rischio di leucemia (WIEMELS, 1999). Insomma, paradossalmente, i bambini "più sani", quelli che non sono mai stati male sono quelli più deboli ed esposti ai pericoli maggiori. Uno studio dimostra che gli adolescenti che da bambini hanno avuto la leucemia risultano avere una immagine di sé più positiva e matura rispetto ai coetanei che non sono passati per questa esperienza: risultano più "forti" e più generosi (MAGGIOLINI ET AL., 2000). Nello stesso modo le famiglie che escono dalla esperienza di un figlio affetto da leucemia, sia con esito positivo che negativo, sembrano più forti, più aperte e generose delle altre.

L'importanza del rischio come componente fondamentale dell'esperienza ludica è stata studiata anche in psicologia (BRUNER, 1976, SUTTON-SMITH, 1997); in etologia (SPINKA, et al. 2001); in filosofia (BENCIVENGA, 1988).
} 
Se si riconosce che invece l'autonomia è importante per lo sviluppo di un bambino, perché possa giocare e fare le necessarie esperienze, gli adulti dovrebbero fare un passo indietro: per un certo periodo ogni giorno, dovrebbero non esserci.

Parlando con i bambini del Consiglio dei bambini di Rosario, in Argentina, su bisogno e sul diritto dei bambini a poter uscire da soli di casa, una bambina di nove anni diceva: "Se mi tengono sempre per mano, poi un giorno dovranno lasciarmela e quel giorno io avrò paura". E un suo compagno proponeva: "Gli adulti dovrebbero aiutarci, però da lontano".

\section{Ricominciamo dai bambini}

I bambini e gli adulti fanno della realtà una diagnosi molto simile. Sia gli uni che gli altri riconoscono che la città è pericolosa, che le automobili sono troppe, che non rispettano i pedoni, che i marciapiedi sono sporchi, mal tenuti e ingombri, che gli attraversamenti pedonali non sono sicuri, che ci sono persone cattive in giro. Ma le conseguenze per gli uni e gli altri sono profondamente diverse.

Gli adulti dicono: "Siccome queste sono le condizioni della città, tu rimani in casa e se devi uscire ti accompagno e ti aspetto".

I bambini dicono: "Siccome queste sono le condizioni della città, bisogna cambiarle".

I bambini non sono rassegnati e non possono accettare la comodità, il possesso di cose, in cambio della loro libertà. D'altra parte i bambini, fin da piccoli, sono capaci di interpretare i propri bisogni e di contribuire al cambiamento delle loro città (HART, 1997). Vale quindi la pena dare loro la parola, chiamarli a partecipare, perché forse in loro nome e per il loro benessere è possibile chiedere ai cittadini adulti quei cambiamenti che difficilmente sono disposti ad accettare e a promuovere per se stessi - pur riconoscendone in teoria l'importanza e l'urgenza.

Dal 1991 il progetto internazionale "La città dei bambini", promosso dall'Istituto di Scienze e Tecnologie della Cognizione (ISTC) del CNR, propone alle Amministrazioni delle città di cambiare il parametro e di passare dall'adulto, maschio, lavoratore e automobilista ai bambini, di abbassare l'ottica ad altezza di bambino per non perdere nessuno ${ }^{6}$. L'assunto di base è semplice ma anche rivoluzionario: una città che cerca di essere adatta ai bambini è una città dove tutti vivranno meglio.

\footnotetext{
${ }^{6}$ Per una migliore conoscenza delle motivazioni, delle proposte e delle esperienze del progetto si possono consultare i volumi Tonucci (1996); Tonucci (2002) e il sito web www.lacittadeibambini.org
} 
Il bambino, esprimendo le sue esigenze, rappresenta bene quelle di tutti i cittadini a partire dai più deboli come i portatori di handicap e gli anziani. Per questo può diventare il paradigma per una nuova filosofia di governo della città.

Il progetto, a cui aderiscono $\mathrm{i}$ sindaci, coinvolge in maniera trasversale l'Amministrazione della città, perché non si tratta di realizzare più strutture o servizi per i bambini, ma di cambiare realmente la città. Oggi fanno parte della sua rete più di sessanta città italiane più alcune città spagnole e le più grandi città argentine. Roma ha aderito al progetto nel 2001 assumendo il ruolo di città capofila.

Il progetto "La città dei bambini” si muove su due assi principali: l'autonomia e la partecipazione dei bambini.

La perdita di autonomia dei bambini è stato probabilmente l'effetto più clamoroso delle trasformazioni degli ultimi decenni nella vita delle città e questo cambiamento ha compromesso la loro possibilità di gioco (HILLMAN, 1993). La restituzione di autonomia ai bambini potrà essere una strada sicura per il recupero e la sopravvivenza delle città. Se i bambini potranno andare a scuola da soli, scendere a giocare con gli amici negli spazi pubblici della città, anche gli anziani, i portatori di handicap e tutti i cittadini potranno vivere di nuovo l'esperienza del passeggio e dell'incontro. Inoltre, una maggiore autonomia avrà anche un impatto specifico sui bambini, attraverso i suoi effetti sulle loro opportunità di gioco: solo se un bambino può uscire di casa, incontrare altri bambini e vivere con loro le esperienze di gioco, senza un diretto controllo degli adulti, il suo gioco avrà la grande capacità di coinvolgerlo completamente e portarlo a grandi conquiste. Solo in queste condizioni il gioco diventa protagonista e il giocattolo solo uno strumento del giocare. Solo in queste condizioni, di fronte all'assenza di strumenti adeguati i bambini procedono alla costruzione dei loro giocattoli utilizzando materiali naturali o recuperando creativamente i materiali rifiutati ${ }^{7}$. Gli scatoloni degli imballaggi, le bottiglie di plastica, i pezzetti di legno, la terra e la sabbia, permettono giochi sempre diversi e di lunga durata. Da tutte le ricerche condotte risulta che l'unico vero antidoto ad un uso eccessivo e acritico della televisione è rappresentato dalla possibilità per i bambini di uscire di casa e di incontrarsi fra di loro. Al contrario è sempre frustrante per l'adulto e per il bambino la rigida applicazione di orari o regole per contingentare l'uso della televisione. Nel momento in cui le famiglie hanno valutato che la città non permette più l'uscita dei bambini di casa si è posto il grande problema di come far passare loro il tempo, di come farsi perdonare questa

7 Si vedano le osservazioni di John Locke, a fine Seicento, sull'opportunità che i bambini, per quanto possibile, si procurino da soli i propri giocattoli, (LOCKE, 1970 pp. 141-143, 175-178, 271-275). 
espropriazione. E, come sempre, la risposta più "adeguata" i genitori l'hanno avuta dalla produzione commerciale che ha aperto prima negozi dedicati esclusivamente ai giocattoli (cinquanta anni fa non esistevano) ed ora veri e propri supermercati del giocattolo (BENCIVENGA, 1995; KLINE, 1995; SUTTON-SMITH, 1986). Ma ha fatto di più, ha messo in vendita i giocattoli, specie per i bambini più piccoli, in farmacia, suggerendo cos' l'idea diabolica di "bambino uguale malato".

Nel progetto "La città dei bambini", la partecipazione dei bambini deve essere cercata e voluta come una corretta accoglienza dell'articolo 12 della Convenzione ONU dei diritti dei bambini che dice che i bambini hanno il diritto di esprimere il loro parere ogni volta che si prendono decisioni che li riguardano, e che il loro parere deve essere tenuto nel giusto conto. Siccome l'Amministrazione di una città prende decisioni che sempre riguardano anche i bambini, occorre trovare le forme adeguate per ottenere il loro parere (TONUCCI; RISSOTTO, 2001).

Esiste una tradizione ormai trentennale, originaria della Francia, che, per far capire ai bambini il funzionamento del Consiglio comunale, propone ai bambini e ai ragazzi di ripeterne la formazione e il funzionamento in una specie di gioco di ruolo nel quale i bambini formano $\mathrm{i}$ partiti, partecipano alle elezioni, eleggono un sindaco e una Giunta che operano secondo il modello adulto. È questa una proposta squisitamente educativa che non interferisce affatto sulle scelte e sul governo della città. La proposta del progetto "La città dei bambini è invece prettamente politica ed evita ogni ipocrisia dei piccoli amministratori, dei piccoli sindaci. Si tratta invece di un gruppo di bambini che dà consigli al sindaco. Per comprenderne il significato valgano le parole del sindaco di Roma Walter Veltroni all'atto dell'apertura del primo Consiglio dei bambini ${ }^{8}$ della città: "Io ho voluto questo Consiglio perché ho bisogno dei vostri consigli, del vostro aiuto. Capita che i grandi si dimentichino di quando erano bambini. Che non ricordino le cose importanti e necessarie per vivere bene questo tempo della vita e che non si ricordino quali siano i sogni, i desideri, le speranze che si hanno quando si ha tutta la vita davanti a sé. Da oggi cominciamo a lavorare insieme perché vogliamo cambiare al città". Da quel momento i bambini hanno cominciato a lavorare per dare consigli al sindaco, per suggerirgli idee, per protestare per quello che non piaceva loro. Da quel giorno hanno cominciato a

\footnotetext{
${ }^{8}$ A Roma si è istituito un Consiglio dei bambini formato da un bambino e da una bambina, uno di quarta e uno di quinta elementare (9-10 anni), di una scuola per ognuno dei 19 Municipi. I consiglieri sono nominati per sorteggio e rimangono in carica per due anni. Il Consiglio si riunisce una volta al mese, in orario scolastico, per l'intera mattinata. Due volte all'anno il Consiglio dei bambini si incontra con il sindaco e con il Consiglio Comunale. Essendo questa l'esperienza che l'ISTC sta seguendo con maggiore impegno, verranno di seguito riferite le proposte di questo Consiglio.
} 
partecipare al governo della città. Da quel giorno sono in difficoltà gli adulti, che spesso non riescono a dare risposte adeguate alle richieste, sempre motivate e precise, dei bambini.

Esistono anche altre proposte perché i cittadini inferiori ai 18 anni pesino sulle decisioni pubbliche. Si parla da tempo per esempio del voto universale e cioè del conferimento del diritto di voto a tutti i cittadini fin dalla nascita. Il voto dei minori verrebbe, secondo queste proposte, esercitato dai genitori, loro legali tutori, $\mathrm{o}$, in alcune proposte, dalle madri, per riequilibrare la partecipazione femminile alla vita pubblica (Philippe Van Pareijs). Questo dibattito nato fra filosofi e studiosi di scienze politiche è giunto in questi ultimi anni a concrete proposte parlamentari per esempio in Germania.

Pur ritenendo interessante questa proposta che inserirebbe nuovi elementi nello stanco dibattito politico (TONUCCI, 1996), ritengo non possa rispondere in maniera soddisfacente al diritto di partecipazione dei bambini sancito dall'articolo 12. Sarebbero infatti ancora gli adulti a prendere le decisioni, anche se a nome dei loro figli. Il Consiglio dei bambini invece potrebbe, se correttamente condotto, raccogliere realmente le opinioni dei più piccoli.

Rimane da chiedersi se sia necessario consultare i bambini se i problemi e gli errori delle città, sono così chiari a tutti gli esperti che se ne occupano sia da un punto di vista psicopedagogico, sia sciale, sia urbanistico. Dall'esperienza di questi quasi quindici anni riteniamo che si possa rispondere affermativamente per almeno tre ordini di ragioni. 1. I bambini sono capaci di far notare problemi che gli adulti tendono a non vedere o a sottostimare, come la mancanza di spazio e di tempo per il gioco, l'assurdo di una eccessiva protezione, l'impossibilità di una mobilità autonoma, ecc. Ascoltando i bambini questi problemi possono assumere quei criteri di urgenza che attualmente sono loro negati. 2. Gli amministratori possono farsi forti di fronte ai loro concittadini delle richieste dei bambini per sollecitare cambiamenti costosi potendo dire "Ce lo chiedono i vostri figli". 3. Per i bambini la partecipazione reale al governo della città diventa una forma forte e diretta di educazione alla democrazia.

\section{Esperienze}

Proviamo ora a ripercorrere il significato dell'autonomia dei bambini e del loro bisogno di giocare attraverso il loro punto di vista, attraverso le proposte e le richieste che i bambini stessi hanno presentato in alcune esperienze di partecipazione infantile al governo delle città.

“Vogliamo da questa città il permesso di uscire di casa”. La precondizione perché il gioco infantile possa esistere è che i bambini possano uscire di casa senza essere accompagnati 
dai genitori. Il Consiglio dei bambini di Roma ha dedicato a questo problema un intero anno di lavoro e nella riunione finale del giugno 2002 che, come di consueto, il Consiglio dei bambini tiene con il Consiglio comunale, con il sindaco e la Giunta, Federico, un consigliere di 11 anni, ha riassunto il lavoro svolto con questa richiesta: "Vogliamo da questa città il permesso di uscire di casa". Una richiesta sorprendente perché un bambino può chiedere questo permesso solo ai suoi genitori e solo loro possono concederlo o negarlo. Ma Federico sapeva che i genitori lo negano "perché la città non lo permette" e allora va dal sindaco e gli dice "allora dammelo tu il permesso di uscire di casa". Da allora la richiesta di Federico è diventato un programma di lavoro per la città di Roma che si interroga su come sia possibile aumentare l'autonomia dei bambini in una città così grande e così complessa. Il problema è importante perché riguarda uno dei temi più sentiti nel dibattito attuale e probabilmente del futuro delle nostre città, quello della sicurezza urbana.

La proposta più comune per risolvere il problema della sicurezza urbana è l'aumento di difesa: aumento di difese individuali dalle porte blindate alle armi personali, aumento di difese sociali dalla polizia alle telecamere nelle strade. Ma queste soluzioni si sono dimostrate sempre inefficaci ed è vero che gli Stati Uniti, che sono il Paese dove si investe di gran lunga di più per la difesa, continua ad essere un Paese altamente insicuro ${ }^{9}$. Di questo erano convinti gli abitanti di alcuni Municipi della Gran Buenos Aires, la grande cintura urbana di 8.000.000 di abitanti che circonda la città di Buenos Aires, che, stanchi delle continue violenze e rapine che subivano i loro bambini, hanno deciso non di chiedere maggiore presenza di polizia, ma maggiore partecipazione degli abitanti dei quartieri per garantire i bambini nei percorsi casa - scuola, secondo le indicazioni del nostro progetto "La città dei bambini" ${ }^{10}$. Sono stati coinvolti i commercianti e gli artigiani, gli anziani e i cittadini perché si ricreasse una vigilanza sociale sui percorsi dei bambini e, secondo le testimonianze dei promotori, gli atti di criminalità nei confronti dei bambini sono crollati del 90\%. La proposta si è diffusa in molti Municipi e si sta affermando anche nella Capitale Federale. Questa iniziativa ripresa in Argentina è la stessa che in molte città italiane si sta proponendo con successo, chiamata "A scuola ci andiamo da soli",

\footnotetext{
9 A fronte delle quasi 3000 vittime del terribile attentato dell'11 settembre nello stesso anno, negli Stati Uniti, perirono 30.000 persone per incidenti causati dall'uso di armi da fuoco (Fonte: MANN, M. L'impero impotente, Piemme, 2004).

10 Clarin, il più diffuso quotidiano argentino, ha dedicato a questa iniziativa diversi articoli. Il 16 luglio 2003, in un articolo intitolato "La gente se une para cuidar a los chicos camino al colegio" (La gente si unisce per proteggere $i$ bambini che vanno a scuola) fa esplicito riferimento al progetto "La città dei bambini", e valuta al $90 \%$ la riduzione di atti criminali dopo che il vicinato, i commercianti e gli anziani si sono mobilitati per garantire sicurezza ai bambini che vanno a scuola nei Municipi di Burzaco, Adrogué, Rafael Calzada, Martinez y Villa Adelina.
} 
che propone ai bambini di scuola elementare, dai sei agli undici anni, di andare a scuola con i loro amici e senza l'accompagnamento di adulti.

Queste esperienze nascondono la convinzione profonda che i bambini per strada fanno sicura la strada. I bambini che vanno a scuola, che giocano con gli amici, ricostruiscono intorno a loro quella preoccupazione e solidarietà dei vicini, degli adulti, che solo apparentemente è scomparsa dalle nostre città. È evidente che se ognuno accompagna il proprio figlio, se i vecchi e i portatori di handicap restano chiusi in casa, tutto si riduce alla dimensione privata e nessuno deve più farsi carico degli altri. Ma se i bambini ricompaiono si costruisce un nuovo vicinato che produce protezione e sicurezza. I bambini sono sicuri e la gente è coinvolta e soddisfatta: "È bello rivedere i bambini che vanno a scuola da soli", mi hanno detto più volte gli abitanti dei quartieri dove si sperimentava con successo l'esperienza "A scuola ci andiamo da soli" (BARALDI; MAGGIONI, 2000; RISOTTO; TONUCCI, 2002; PREZZA ET. AL., 2010; SHAW ET AL., 2013).

Se la sicurezza e quindi la possibilità di uscire è una precondizione del giocare infantile esistono poi due condizioni necessarie che sono venute a mancare e che i bambini richiedono con chiarezza: Tempo libero e Spazio pubblico.

Un tempo libero per giocare. Con i bambini di Florencio Varela, in Argentina, abbiamo discusso se i due articoli della Convenzione dei diritti dei bambini del 1989, il 28 che afferma il diritto all'istruzione e il 31 che afferma il diritto al gioco avessero nella loro vita di tutti i giorni un uguale peso. Si dovrebbe infatti ritenere che, essendo due articoli della stessa legge, debbano avere uguale dignità, uguale rispetto e uguale considerazione. Naturalmente i bambini hanno detto che non è così, che il gioco non è considerato importante dagli adulti e che spesso non hanno tempo per giocare, mentre non succede mai che possano non avere tempo per andare a scuola o per fare i compiti. Anzi, secondo loro il diritto di andare a scuola lo sentivano principalmente come un dovere e il diritto al gioco come qualcosa che gli adulti considerano tempo perso. Abbiamo allora immaginato che anche il diritto al gioco debba essere considerato un dovere e abbiamo definito quante ore dovrebbe giocare ogni giorno un bambino per assolvere a questo dovere. Li divertiva molto l'idea che loro potessero andare a scuola un'ora più tardi o non andarci del tutto se dovevano recuperare delle ore che non avevano potuto dedicare al gioco il pomeriggio precedente, perché impegnati per esempio nei compiti o nell'accompagnare i genitori al supermercato. Posto in questo modo il problema sembra effettivamente un paradosso, ma se sono vere le considerazioni iniziali sulla importanza del gioco si dovrebbe considerare questa proposta in modo molto più serio. Considerarla un 
doveroso investimento per la società. Ma non un investimento per il futuro, come di solito si pensa, ma per il presente: i bambini che giocano nella città producono dei cambiamenti e dei miglioramenti da oggi, da subito.

I bambini del Consiglio dei bambini di Roma, avendo fatto lo stesso percorso dei loro colleghi argentini, hanno coinvolto i loro compagni di scuola in una indagine sui problemi che i bambini hanno rispetto al gioco. I problema più forte è risultato la mancanza di tempo e la proposta più citata per aumentare il tempo di gioco è stata di avere meno compiti per casa. Il Consiglio dei bambini ha allora scritto una lettera aperta a tutte le scuole elementari di Roma per chiedere ai loro insegnanti di non avere più compiti per casa durante $\mathrm{i}$ fine settimana $\mathrm{e}$ durante le vacanze. I bambini propongono di essere disposti a fare, in quei periodi, alcune attività, ma solo quelle desiderate e senza controlli. I bambini non sono pedagogisti e non si esprimono sulla validità dei compiti e sulla correttezza o meno di darli. Considerano solo che la scuola non può occupare tutto il loro tempo e chiedono che una parte, peraltro limitata, venga loro restituita per poter giocare. Silvia comunque, consigliera di 10 anni, avanza una personale valutazione: "Con i compiti non si sviluppa la fantasia, con il gioco invece sì". È sorprendente che diversi risultati scientifici confermano esattamente la tesi di Silvia.

La richiesta dei bambini romani ha avuto una grande risonanza nei mezzi di comunicazione, sia locali che nazionali, molti esperti si sono espressi a loro favore ma purtroppo in poche scuole si sono cambiate le vecchie abitudini. E invece la scuola dovrebbe essere fortemente interessata a che gli alunni possano dedicare le ore pomeridiane, del fine settimana e delle vacanze a vivere esperienze interessanti di esplorazione e di gioco con i loro compagni perché queste esperienze potranno essere un alimento fondamentale per il lavoro scolastico. In assenza di questo contributo degli alunni la scuola sarà costretta a tornare ai suoi programmi e ai suoi libri di testo, tornando ad essere una cattiva scuola.

Uno spazio pubblico per giocare. La seconda condizione necessaria per poter giocare, una volta che si possa uscire di casa senza controllo e che si abbia il tempo libero per farlo, è avere posti dove andare. Già si è detto di come i giardinetti dedicati al gioco infantile non siano luoghi adatti per una attività così importante come il gioco. Per giocare i bambini hanno bisogno di uno spazio che cresca con le loro capacità, le loro autonomie e le loro competenze. Uno spazio che sappia accompagnare il loro sviluppo, che sappia offrire esperienze nuove, nuove scoperte, muove ricchezze. Ai bambini per crescere e per giocare serve la città, né più né meno.

A questo proposito i bambini del Consiglio dei bambini di Roma hanno fatto una scoperta inquietante: nel Regolamento di Polizia Urbana l'articolo 6 recita: "E' vietato 
qualunque gioco sul suolo pubblico". Ritenendo che questo articolo fosse in contrasto con l'articolo 31 della Convenzione dei diritti dei bambini, che dal 1991 è legge nazionale italiana, hanno scritto al sindaco chiedendogli di modificare quell'articolo.

Il sindaco ha risposto ai bambini riconoscendo corretta la loro protesta e promettendo di cambiare l'articolo 6 del Regolamento. Dopo questa vittoria i bambini del Consiglio hanno fatto un altro passo e hanno scritto una lettera alle assemblee di condominio di tutta la città invitando i condòmini a rivedere i regolamenti condominiali che spesso limitano o proibiscono il gioco dei bambini. Il sindaco si è dichiarato favorevole anche a questa seconda richiesta dei bambini e potrebbe accompagnarla con un suo personale invito ai cittadini romani. La protesta dei bambini romani potrebbe quindi avere importanti conseguenze: far sparire dai luoghi pubblici della città i cartelli di divieto di gioco, aprire un dibattito in tutta la città sul diritto dei bambini a giocare negli spazi comuni delle loro case e dei loro condomini e, speriamo, provocare un simile fenomeno in altre città che quasi sempre hanno articoli di Regolamento di Polizia Urbana simili all'articolo 6 di Roma.

Più gioco per tutti. Nel 1998 il Consiglio dei bambini del distretto Nord della città argentina di Rosario, durante una riunione straordinaria del Consiglio comunale al quale partecipavano decine di bambini in rappresentanza delle scuole della città, chiese l'istituzione del "Giorno del gioco" come appuntamento annuale con le seguenti caratteristiche: cadrà ogni anno il primo mercoledì di ottobre; le scuole saranno aperte solo per giocare; gli impiegati pubblici avranno un'ora pagata per giocare; alcune strade della città saranno chiuse al traffico. Vale la pena esaminare queste condizioni che contengono elementi fortemente innovativi e che, come spesso avviene, superano il puro interesse dei bambini stessi. 1. La giornata richiesta si chiamerà "Giorno del gioco" e non "Giorno dei bambini". Nelle discussioni che il Consiglio dei bambini dedicò a questa importante proposta l'analisi dei bambini riguardava non solo la loro categoria ma l'intera cittadinanza. A loro avviso gli adulti giocano poco o non giocano più e questo crea molti problemi, sia agli adulti che ai bambini. "Se gli adulti giocano di più sono migliori", dicevano. La proposta è quindi di avere un giorno all'anno in cui tutti sono invitati a giocare. 2. Il giorno dovrà essere un lavorativo, non per guadagnare un giorno di vacanza, ma per rompere lo schema adulto del lavoro separato dal piacere, dal gioco, dal rapporto con gli altri. Dovrà essere lavorativo in modo che le scuole siano aperte, ma aperte solo per giocare: docenti, genitori e bambini dovranno preparare questa giornata speciale. È divertente pensare che una volta all'anno a scuola si vada solo per piacere e probabilmente questa "sgrammaticatura" può far molto bene alla scuola stessa. 3. I lavoratori pubblici dovranno avere 
un permesso speciale di un'ora da dedicare al gioco, per giocare loro stessi e per giocare con i bambini. Anche in questo caso, uffici pubblici e servizi dovranno prepararsi a questo strano incontro con i bambini in modo da proporre loro qualche forma di gioco, possibilmente collegata al loro lavoro, al loro ruolo. 4. Alcune strade saranno chiuse per diventare luogo di gioco. Questa ultima condizione, ormai comune nelle nostre città, che in una giornata particolare (di solito una domenica) chiudono tutte o alcune strade per lasciarle al gioco dei bambini, in questa esperienza acquista un valore maggiore in un giorno lavorativo: gli adulti liberati dal lavoro potranno realizzare sulle strade alcuni giochi con i bambini della città.

Il Consiglio comunale approvò la richiesta all'unanimità.

Da venti anni il "Giorno del gioco" si celebra a Rosario e ogni anno aumenta la partecipazione degli adulti. Il permesso di lasciare il lavoro, che originariamente era stato chiesto dai bambini per i lavoratori comunali (perché il Comune poteva deliberare solo in merito ai suoi dipendenti), è stato concesso da più di 300 enti, fra cui le Poste, le banche, esercizi pubblici e privati. In ognuno di questi luoghi di lavoro sono stati preparati giochi che coinvolgevano adulti e bambini. Che cose di questo genere succedano in una società come la nostra, apparentemente interessata solo al profitto, ai consumi e chiusa a qualsiasi proposta culturale e solidale, apre legittimi spiragli alla speranza.

Nel 2014 il Consiglio diei bambini di Buenos Aires ha proposto che si celebrasse "La notte dei bambini" e da cinque anni i bambini di molti quartieri della città vivono esperienze di esplorazione e di gioco dalle 21 e dopo le 23 si ritrovano tutti insieme in uno stadio, al buio, ciascuno con una pila in mano, per un incontro di festa, di canti, di balli.

\section{Conclusioni}

È come se la città si fosse dimenticata dei bambini. Ha fatto moltissimo per le automobili, molto per gli adulti. Sembra che abbia fatto molto anche per i bambini attraverso i servizi per l'infanzia, ma nidi, scuole dell'infanzia, giardinetti, parchi gioco, ludoteche, sono tutti spazi pensati più per i genitori che non sanno dove lasciare i figli che per rispondere alle reali esigenze dei bambini. Di fatto in questa città i bambini non possono svolgere la loro attività più importante, il loro vero lavoro, l'esperienza che condizionerà più di ogni altra il loro futuro: giocare.

Una città non può assumersi questa grave responsabilità. Non può negare ai suoi cittadini la possibilità di godere dei loro diritti, almeno di quelli definiti e consacrati dalle leggi 
e il diritto di gioco è sancito dall'articolo 31 della Convenzione internazionale dei diritti dei bambini.

Una città potrà permettere ai suoi cittadini più piccoli di giocare se saprà restituire la loro funzione originale ai suoi spazi pubblici. Le strade, i marciapiedi, le piazze e i giardini, dovranno tornare ad essere i luoghi dell'incontro e dello scambio, del passeggio. E se saprà farlo la città dovranno farlo anche i cittadini, restituendo all'uso pubblico le scale, gli androni e i cortili dei condomini. In quegli spazi i bambini potranno tornare a giocare, incontrandosi con gli adulti e in particolare con gli anziani, che potranno tornare ad uscire di casa.

Sul Vesuvio, il vulcano di Napoli, mi hanno fatto conoscere un lichene che riesce a colonizzare la pietra lavica, la durissima pietra che copre le pendici del monte dopo una eruzione. Questa piantina riesce a penetrare nella lava, a frantumarla e a trasformarla lentamente in terreno fertile, dove potranno crescere le viti che producono rinomati vini vesuviani.

I bambini possono essere i licheni delle nostre città: con la loro presenza, con i loro giochi, invadendo gli spazi pubblici, sono capaci di modificare gli assurdi e irresponsabili comportamenti di noi adulti, obbligarci ad essere più prudenti con le auto, ad essere più attenti a quello che ci succede intorno, a rispettare di più l'ambiente dove viviamo e dove vivranno $\mathrm{i}$ nostri figli e i nostri nipoti.

\section{RIFERIMENTI}

ADER, J., JOUVE, H., 'Jeu et contexte urbain'. Architecture \& Comportement 7, 1991. p.115-119.

BARALDI, C., MAGGIONI, G.(eds.). Una città con i bambini, progetti ed esperienze del Laboratorio di Fano, Roma: Donzelli Editore, 2000.

BATESON, G., The Message “This Is Play”, New York: Josiah Macy Jr. Foundation, 1956.

BAUMAN, Z., The individualized society, Cambridge: Polity Press, 2001.

BENCIVENGA, E. Giocare per forza. Critica alla società del divertimento, Milano: Mondadori, 1995.

BENCIVENGA, E. Tre dialoghi, Torino: Bollati Boringhieri, 1988.

BLAKELY, K. S. 'Parents' conceptions of social dangers to children in the urban environment' Children's Environments 11, 1994. p. 16-25.

BOZZO, L. 'Il gioco e la città', Paesaggio urbano 2, 1995, p. 30-33. 
BRUNER, J. S. 'Nature and uses of immaturity', in J. S. Bruner, A. Jolly, K. Silva (eds.), Play. Its role in development and evolution, New York: Basic Books, 1976.

CAILLOIS, R. Les jeux et les hommes, Paris: Gallimard, 1967.

CHAWLA, L. Growing up in an urbanizing world, Paris/London: Unesco Publishing/Earthscan, 2001.

DE SAINT-EXUPÉRY, A. Le Petit Prince, Paris: Gallimard, 1943.

GARLING, T., VALSINER, J. (eds.): Children within environment: Toward a psychology of accident prevention, New York: Plenum Press, 1985.

GARVEY, C. Play, Cambridge: Harvard University Press, 1977.

GERMANOS, D. 'La relation de l'enfant à l'espace urbain: perspectives educatives et culturelles', Architecture \& Comportement 2, 1995, p. 54-63.

GOMBRICH, E. H. Meditations on a hobby horse and other essays on the theory of art, London: Phaidon, 1971.

HART, R. Children's Participation: The Theory and Practice of Involving Young Citizens in Community Development and Environmental Care, London: Earthscan Publication Limited, 1997.

HILLMAN, M. (ed.). Children, transport and the quality of life, London: Policy Studies Institute, 1993.

HUIZINGA, J. Homo Ludens, Amsterdam: Panteon Verlag, 1939.

KLINE, S. 'The promotion and marketing of toys: Time to rethink the paradox?'. In: PELLEGRINI, A. The future of play theory, Albany: State of New York University Press, 1995.

LOCKE, J. Some thoughts concerning education, Indianapolis/Cambridge: Hackett Publishing Company (orig. ed. 1693), 1996.

LYNCH, K. 'The Spatial World of the child', in W. Michelson, S.V. Levine, E. Michelson (eds.), The child in the city: Today and tomorrow, Toronto: Univerity of Toronto Press.

MANN, M. Incoherent empire, London: Verso Books, 1979/2003.

MARILLAUD, J. 'Jeu et securité dans l'espace pubblic', Architecture \& Comportement 7, 1991, p. 137-145.

MUMFORD, L. 'La pianificazione per le diverse fasi della vita', Urbanistica 1, 1945, p. 7-11.

PIAGET, J. La formation du symbole chez l'enfant, Neuchâtel: Delachaux et Niestlé, 1945.

PREZZA, M., ALPARONE, F. R., RENZI, D., PIETROBONO, A. Social Participation and Independent Mobility in Children: The Effects of Two Implementations of "We Go to School Alone', Journal of Prevention \& Intervention in the Community, 38, 2010, p. 8-25. 
PREZZA, M., PILlONI, S., MORABITO, C., SERSANTE, C., ALPARONE, F. R. 'La mobilità autonoma dei bambini nel contesto urbano e il cortile, il parco e la strada privata come spazi di gioco', Psicologia della salute 3, 2000, p. 81-97.

RISSOTTO, A., TONUCCI, F. 'Freedom of Movement and Environmental Knowledge in Elementary School Children', Journal of Environmental Psychology 22, 2002, p. 65-77.

SHAW, B., WATSON, B., FRAUENDIENST, B., REDECKER, A., JONES, T., HILLMAN, M. Children's independent mobility: a comparative study in England and Germany (1971 2010), London: Policy Studies Institute, 2013.

SPINKA, M., NEWBERRY, R. C., BEKOFF, M. 'Mammalian play: Training for the unexpected', Quarterly Review of Biology 76, 2001, p. 141-168.

SUTTON-SMITH, B. The ambiguity of play, Cambridge: Cambridge University Press, 1997.

SUTTON-SMITH, B. Toys as culture, New York: Gardner Press, 1986.

TAYLOR, A . F., WILEY, A., KUO, F. E., SULLIVAN, W. C. 'Growing up in the Inner City: Green Spaces as Places to Grow’ Environment and Behavior 30, 1998, p. 3-27.

TONUCCI, F. La città dei bambini, Bari: Laterza, 1996.

TONUCCI, F. Se i bambini dicono: adesso basta!, Bari: Laterza, 2002.

TONUCCI, F., RISSOTTO, A., 'Why Do We Need Children's Participation? The Importance of Children's Participation in Changing The City', Journal of Community and Applied Social Psychology 11, 2001, p. 407-419.

VYGOTSKY, L. S. 'Play and its role in the mental development of the child', Soviet Psychology 12, VI, 1966.

\section{SOBRE O AUTOR:}

\section{Francesco Tonucci (FRATO)}

Istituto di Scienze e Tecnologie della Cognizione - ISTC - Consiglio Nazionale delle Ricerche, Itália. E-mail: francesco.tonucci@istc.cnr.it

iD http://orcid.org/0000-0003-1406-7997 